\title{
Cosmic-Ray Spectra in Interstellar Space
}

\author{
R. A. Mewaldt ${ }^{\mathrm{a}}$, M. E. Wiedenbeck ${ }^{\mathrm{b}}$, L. M. Scott ${ }^{\mathrm{c}}$, W. R. Binns ${ }^{\mathrm{c}}$, \\ A. C. Cummings ${ }^{\mathrm{a}}$, A. J. Davis ${ }^{\mathrm{a}}$, M. H. Israel ${ }^{\mathrm{c}}$, R. A. Leske ${ }^{\mathrm{a}}$, \\ E. C. Stone ${ }^{\mathrm{a}}$, and T. T. von Rosenvinge ${ }^{\mathrm{d}}$ \\ ${ }^{a}$ California Institute of Technology, Pasadena, CA 91126, USA \\ ${ }^{b}$ Jet Propulsion Laboratory, California Institute of Technology, Pasadena CA 91190, USA \\ ${ }^{c}$ Department of Physics, Washington University, St. Louis MO. 63105. USA \\ ${ }^{d}$ NASA/Goddard Space Flight Center, Greenbelt MD 20771, USA
}

\begin{abstract}
At energies below $\sim 300 \mathrm{MeV} /$ nuc our knowledge of cosmic-ray spectra outside the heliosphere is obscured by the energy loss that cosmic rays experience during transport through the heliosphere into the inner solar system. This paper compares measurements of secondary electron-capture isotope abundances and cosmic-ray spectra from ACE with a simple model of interstellar propagation and solar modulation in order to place limits on the range of interstellar spectra that are compatible with both sets of data.
\end{abstract}

\section{INTRODUCTION}

Among the most important clues to the nature of Galactic cosmic-ray sources and accelerators are measurements of cosmic-ray energy spectra. There are now highresolution measurements of the spectra of almost all of the elements from $\mathrm{H}$ to $\mathrm{Ni}$ at 1 $\mathrm{AU}$, but to relate these to the spectrum of cosmic rays accelerated in the cosmic-ray source(s) it is necessary to take into account significant changes that take place during cosmic-ray transport through the interstellar medium and the heliosphere.

It is generally believed that most galactic cosmic rays are accelerated by supernova shock waves traveling through the interstellar medium (ISM) [1, 2], leading to "source spectra" of the form $d J / d T \propto P^{-(2+\delta)}$, where $T$ is kinetic energy/nuc, $P$ is momentum/nuc and $\delta$ depends on the Mach number of the strongest shocks encountered. At energies above a few $\mathrm{GeV} /$ nuc, the spectra of "primary" species (e.g., $\mathrm{H}, \mathrm{He}, \mathrm{C}, \mathrm{O}, \mathrm{Ne}, \mathrm{Mg}, \mathrm{Si}$, and $\mathrm{Fe}$ ) all have observed slopes of $d J / d T \propto T^{-2.75}$. In contrast, "secondary" species that are rare in the source material (e.g., Li, Be, B, and products of $\mathrm{Fe}$ fragmentation such as $\mathrm{Sc}, \mathrm{Ti}$, and $\mathrm{V}$ ) all have softer spectra, and secondary/primary ratios such as $\mathrm{B} / \mathrm{C}$ and $(\mathrm{Sc}+\mathrm{Ti}+\mathrm{V}) / \mathrm{Fe}$ decrease with increasing energy due to rigidity-dependent escape from the Galaxy. The cosmic-ray transport/solar-modulation model used in this study assumes source spectra proportional to $P^{-2.35}$ for all primary species and a rigidity-dependent mean free path for escape from the Galaxy [3, 4] of a form suggested by Soutoul and Ptuskin [5].

At energies below a few hundred $\mathrm{MeV} / \mathrm{nuc}$ our knowledge of interstellar cosmicray spectra is obscured by the heliosphere - low-energy cosmic rays are to a large extent prevented from entering the inner heliosphere by the interplanetary magnetic 
field, and a considerable range of interstellar spectral shapes can be shown to be consistent with 1-AU observations with appropriate choices for the interplanetary diffusion coefficient. In addition, because cosmic rays lose at least several hundred $\mathrm{MeV} /$ nuc as they traverse the inner heliosphere [6], we have essentially no information on the interstellar spectra of cosmic rays below $\sim 200$ to $\sim 300 \mathrm{MeV} / \mathrm{nuc}$.

It is hoped that the Voyagers, or an Interstellar Probe, will eventually escape the cosmic-ray modulation region and measure interstellar cosmic-ray spectra directly. However, it has recently become clear that a considerable fraction of "cosmic-ray modulation" occurs in the heliosheath, well beyond the present location of the Voyagers [7, 8, 9, 10]. A comparison of interstellar spectra used in several recent studies is shown in Figure 2, along with solar-minimum data from ACE and Voyager. For these interstellar spectra to be consistent with the observations, modulation in the heliosheath must be between $\sim 1.5$ and $\sim 3$ times that between 1 AU and 70 AU.

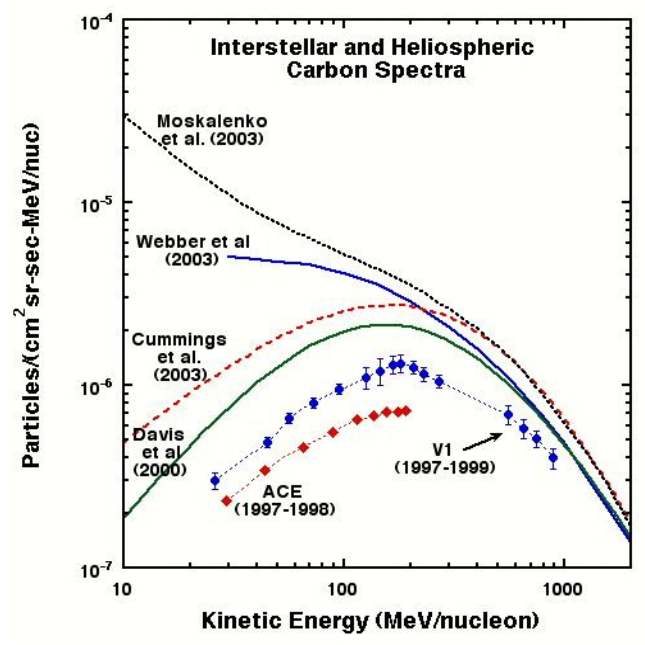

FIGURE 1. Comparison of local interstellar carbon spectra used in several recent studies [11, 12, 13, 14]. Also shown are solar minimum carbon data from ACE at $1 \mathrm{AU}$ and Voyager-1 at 70 AU [13].

Recently, a new probe of this energy-loss process was demonstrated that is based on studies of the energy spectra of the secondary radioactive isotopes ${ }^{49} \mathrm{~V}$ and ${ }^{51} \mathrm{Cr}$, which decay only by electron capture (EC). In cosmic rays the abundances of EC isotopes is related to the probability of attaching an electron from the IS gas: at low energies attachment and decay is much more likely. In a recent paper Niebur et al. [15] used isotope observations from ACE to provide direct evidence that the amount of energy loss increases by 400 to $700 \mathrm{MV}$ ( 200 to $300 \mathrm{MeV} / \mathrm{nuc}$ ) from solar minimum to solar maximum (1997-1998 to 2000-2001). In this paper we extend the work of Niebur et al. and investigate the range of interstellar spectra that is consistent with solar-cycle observations of both the ${ }^{51} \mathrm{~V} /{ }^{51} \mathrm{Cr}$ ratio and the Fe spectrum at $1 \mathrm{AU}$ (note that ${ }^{51} \mathrm{~V}$ is the daughter of ${ }^{51} \mathrm{Cr}$ decay, and that $\mathrm{Fe}$ fragmentation produces most of the $\mathrm{Ti}, \mathrm{V}$, and $\mathrm{Cr}$ observed in cosmic rays). 


\section{APPROACH}

Niebur et al. [15] used a cosmic-ray transport and solar-modulation model [4, 3], which we also adopt here. The model assumes that primary cosmic rays are accelerated to produce source spectra $d J / d T \propto P^{-2.35}$. In addition, cosmic-ray sources are assumed to be uniformly distributed in space and time, with a rigidity-dependent mean free path for leakage from the Galaxy $[3,5]$ and a uniform interstellar density of $0.3 \mathrm{H}$ atoms $/ \mathrm{cm}^{3}$, based on observations of four radioactive clocks [4]. Nuclear fragmentation, ionization energy loss, radioactive decay, and electron-attachment and stripping are taken into account. The first stage of the model results in interstellar spectra for species from $\mathrm{Be}$ to $\mathrm{Ni}$, which then serve as input to a spherically-symmetric solar modulation model that includes diffusion, convection, and adiabatic energy loss.

It is common to characterize the solar modulation level over the solar cycle by the "modulation parameter", $\phi,[16]$, where

$$
\phi \propto \int\left(V_{\mathrm{sw}} / \kappa\right) d r \quad(\text { in } \mathrm{MV}) .
$$

Here $V_{\mathrm{sw}}$ is the solar wind speed, $\kappa$ is the diffusion coefficient, and the integral extends from $1 \mathrm{AU}$ to the boundary of the modulation region $\left(R_{b}\right)$. In practice, many $\kappa, V_{\mathrm{sw}}$ and $R_{b}$ combinations have the same effect. There is also a wide range of interstellar spectra that can produce the same intensity level at $1 \mathrm{AU}$ with a judicious choice of $\kappa$, including the spectra in Figure 1. In this paper we will attempt to narrow the range of possible interstellar spectra by requiring agreement with EC isotope data.

Calculations for the $P^{-2.35}$ source spectra considered by Niebur et al. [15] are compared with spectral and isotope data from ACE in Figure 2. Note that the $\mathrm{Fe}$ spectra at solar minimum require $\phi \approx 400 \mathrm{MV}$, while solar-maximum Fe requires $\phi \approx$ 800 to $1000 \mathrm{MV}$. The right hand panel shows that the solar minimum ${ }^{51} \mathrm{~V} /{ }^{51} \mathrm{Cr}$ ratio is consistent with $\phi \approx 400 \mathrm{MV}$. Niebur et al. also used the ${ }^{49} \mathrm{Ti} /{ }^{49} \mathrm{~V}$ ratio in their study; in this paper we consider only the ${ }^{51} \mathrm{Cr}$ to ${ }^{51} \mathrm{~V}$ decay.
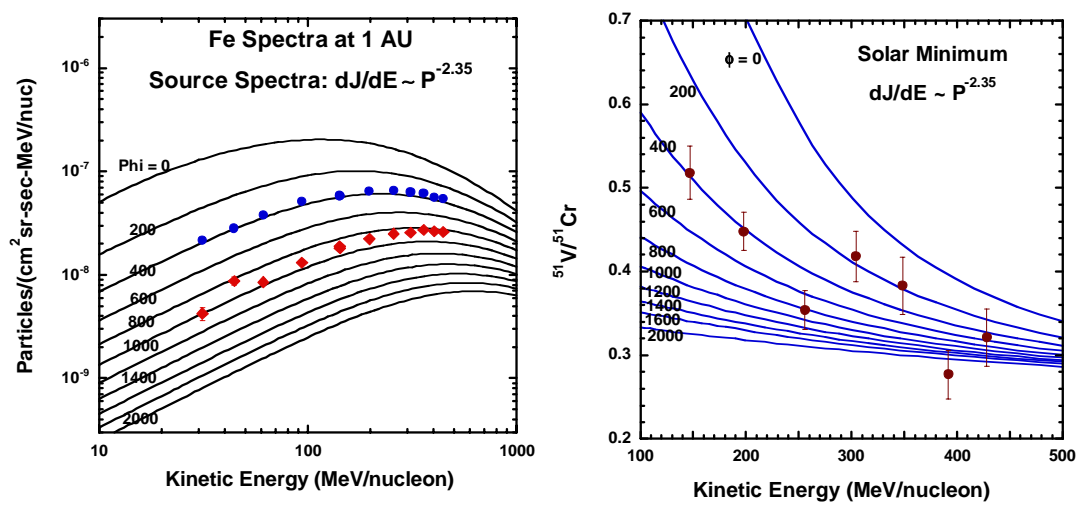

FIGURE 2. The results of the model calculations for $P^{-2.35}$ source spectra (solid lines; labels by solar modulation level) are compared with measurements from ACE. The left panel shows solar minimum and maximum Fe spectra (labeled by the modulation parameter $\phi$ ), while the right panel shows the ${ }^{51} \mathrm{~V} /{ }^{51} \mathrm{Cr}$ ratio. 
In order to study a range of possible interstellar spectra, we consider source spectra of the form $d J / d T=C_{i}\left(T+T_{o}\right)^{-2.35}[17,18]$ where $C_{i}$ depends on species, and $T_{o}$ is independent of species. We consider $T_{o}$ values from 200 to $1000 \mathrm{MeV} /$ nuc (Figure 3), including spectral shapes both softer and harder than $P^{-2.35}$. Complete transport and solar-modulation calculations were performed for source spectra with $T_{o}=200,400$, 600, 800, and $1000 \mathrm{MeV} /$ nuc. Interstellar spectra for ${ }^{51} \mathrm{~V},{ }^{51} \mathrm{Cr}$, and $\mathrm{Fe}$ were used to calculate 1-AU spectra for modulation parameters $\phi$ from 0 to $2000 \mathrm{MV}$ in steps of 50 MV.

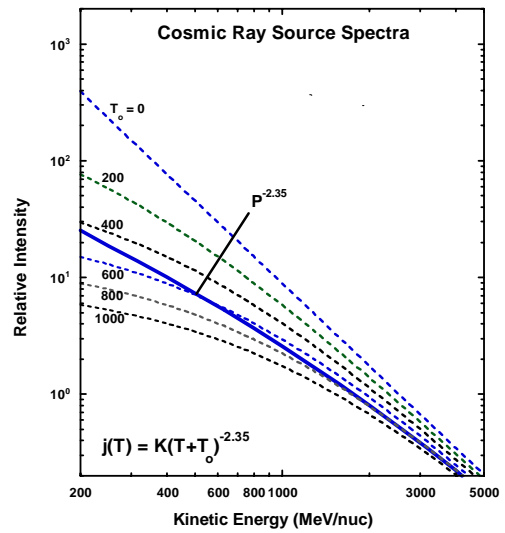

FIGURE 3. Comparison of the source spectra considered in this study.
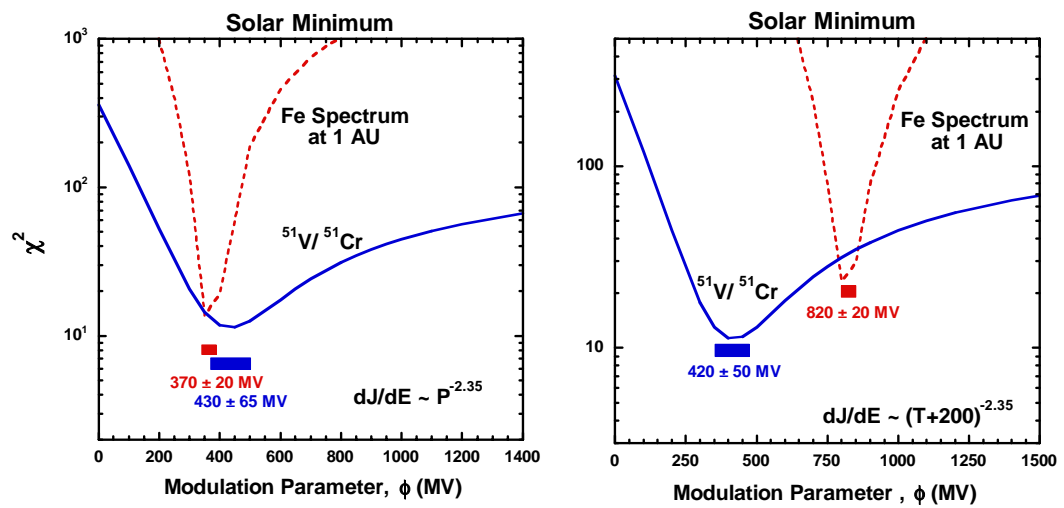

FIGURE 4. Plots of $\chi^{2}$ vs. the modulation parameter $\phi$ for $P^{-2.35}$ and $(T+200)^{-2.35}$ source spectra at solar minimum, including both the $\mathrm{Fe}$ intensity and ${ }^{51} \mathrm{~V} /{ }^{51} \mathrm{Cr}$ ratio. The bars indicate the range of $\phi$ over which $\chi^{2}$ varies from a minimum to $\chi^{2}+1$. There are ten degrees of freedom for the Fe spectra evaluations and six degrees of freedom for ${ }^{51} \mathrm{~V} /{ }^{51} \mathrm{Cr}$. For $P^{-2.35}$ source spectra there is consistency between the $\chi^{2}$ minima; for $(T+200)^{-2.35}$ source spectra it is not possible to fit both with the same $\phi$ value.

The calculated Fe intensity and ${ }^{51} \mathrm{~V} /{ }^{51} \mathrm{Cr}$ ratios at $1 \mathrm{AU}$ were compared to ACE measurements at both solar minimum and maximum and a $\chi^{2}$ goodness of fit was 
evaluated for each. For the Fe spectra we used data from both the SIS and CRIS instruments on ACE; the isotope data are from CRIS (5). In Figure 4 we show how the $\chi^{2}$ values vary with the choice of $\phi$ for $P^{-2.35}$ and $(T+200)^{-2.35}$ spectra at solar minimum. Comparisons were made for $d J / d T \propto\left(T+T_{o}\right)^{-2.35}$ spectra with $T_{o}$ ranging from 200 to $1000 \mathrm{MeV} /$ nuc. The goodness of fit was evaluated for the Fe-spectra and EC-isotope data at both solar minimum and maximum. Figure 4 illustrates an example where the Fe and EC-isotope results are consistent and one where they are not. Figure 5 summarizes the $\phi$ and $T_{o}$ values that minimize $\chi^{2}$, and indicates a range allowed by $\chi^{2}+1$ that is centered on $T_{o}=575 \pm 25 \mathrm{MeV} /$ nuc. The $\chi^{2}+4$ limits $(2 \sigma)$ give an allowable range $T_{o}=410$ to $630 \mathrm{MeV} /$ nuc. Note that the solar-minimum comparison places tighter restrictions on the IS spectra, as would be expected.
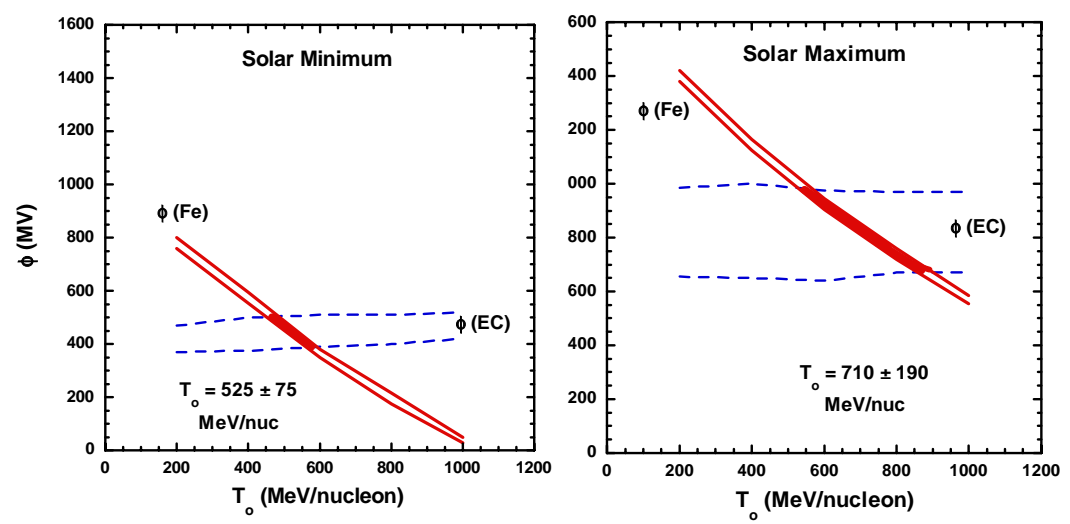

FIGURE 5. Value of $\phi$ for which $\chi^{2}$ is a minimum are plotted vs. the spectral parameter $T_{o}$. The left panel includes solar-minimum comparisons with the Fe intensity and ${ }^{51} \mathrm{~V} /{ }^{51} \mathrm{Cr}$ ratios; the right panel is for solar maximum. Values of $T_{o}$ compatible with both the Fe intensity and ${ }^{51} \mathrm{~V} /{ }^{51} \mathrm{Cr}$ ratio are indicated.

\section{DISCUSSION}

The range of $T_{o}$ values compatible with both the $\mathrm{Fe}$ spectra and ${ }^{51} \mathrm{~V} /{ }^{51} \mathrm{Cr}$ measurements $\left(T_{o}=575 \pm 25 \mathrm{MeV} /\right.$ nuc $)$ is quite narrow. The range of allowed spectral shapes (refer to Figure 3 ) is consistent with and roughly centered on the $P^{-2.35}$ spectra used by Niebur et al. [15], supporting this choice because it provides a selfconsistent interpretation of the spectral and EC isotope data. We are now in the process of evaluating the other IS spectra in Figure 1 to see whether the $\phi$ level required to fit the solar-minimum $C$ data at $1 \mathrm{AU}$ is consistent with the $\phi \approx 430 \pm 70$ $\mathrm{MV}$ range that we find for the solar-minimum ${ }^{51} \mathrm{~V} /{ }^{51} \mathrm{Cr}$ data (see Figure 5). It is clear that interstellar spectral shapes significantly steeper than that of Moskalenko et al. (Figure 1) are not allowed.

Two caveats should be considered. The steady-state propagation model used here assumes cosmic-ray sources uniform in space and time, such that the observed cosmic rays have traversed a broad distribution of interstellar pathlengths. However, the solar system is embedded within a low-density $\left(\sim 10^{-3} \mathrm{H}\right.$ atoms $\left./ \mathrm{cm}^{3}\right)$ region (our "Local 
Bubble"), within which 10-20 supernovae have exploded over the past 10-20 MY [19]. If a significant fraction of the observed cosmic rays have passed through very little matter without contributing their share of $\mathrm{V}$ and $\mathrm{Cr}$ secondaries, the assumptions of the model are not satisfied. For example, Davis et al. [3] and Moskalenko et al. [14] have considered models in which cosmic rays that originate within our Local Bubble contribute $\sim 20 \%$ of the intensity of primary species such as Fe at $\sim 300$ $\mathrm{MeV} /$ nuc without contributing significantly to the production of $\mathrm{Ti}, \mathrm{V}$, and $\mathrm{Cr}$. However, this study has shown that changing the spectral shape or intensity of Fe has little effect on the IS ${ }^{51} \mathrm{~V} /{ }^{51} \mathrm{Cr}$ ratio at a given energy. Therefore, it should still be possible to test whether these two-component models can satisfy the spectral and EC isotope data at $1 \mathrm{AU}$ over the solar cycle with self-consistent solar-modulation levels.

A second consideration concerns the geometry of the modulation region in the heliosheath. The modulation model used here assumes a standard sphericallysymmetric geometry in computing adiabatic energy-loss. It does not include the effects of drifts. While we consider it unlikely that the inclusion of drift effects will affect these results significantly, it will be important to evaluate if comparable energy losses occur in models with improved representations of modulation processes in the heliosheath.

\section{ACKNOWLEDGMENTS}

This work was supported by NASA at Caltech (under grants NAG5-6912 and NAG5-12929), JPL, Washington University, and Goddard Space Flight Center.

\section{REFERENCES}

1. Blanford, R. D., and Ostriker, J. P., Astrophys. J., 237, 793 (1980).

2. Axford. W. I., Proc. $17^{\text {th }}$ Int. Cosmic Ray Conf, 12, 155 (1981).

3. Davis, A. J., et al., in Acceleration and Transport of Energetic Particles in the Heliosphere, AIP Conf. Proc. 528, ed. R. A. Mewaldt et al., (New York, AIP), pp. 421-424, (2002).

4. Yanasak, N. E., et al. Astrophys. J., 563, 768-792 (2001).

5. Soutoul, A., and Ptuskin, V., Proc. $26^{\text {th }}$ Int. Cosmic Ray Conf., 4, 184 (1999).

6. Goldstein, M. L., Fisk, L. A., and Ramaty, R., Phys. Rev. Lett., 25, 832 (1970).

7. McDonald, F. B., Heikila, B, Lal, N., and Stone, E. C., J. Geophys. Res., 105, 108, (2000).

8. Webber, W. R., and Lockwood, J. A., J. Geophys. Res., 102, 9773-9779, 1997.

9. Langner, U. W., Potgieter, M. S., and W. R. Webber, J. Geophys. Res., 108, No. A10, 8039, doi:10.1029 / 2003JA009934 (2003).

10. Florinski, V., Zank, G. P., and N. V. Pogorev, J. Geophys. Res., 108, No. A6, 1228, doi:10.1029 / 2002JA009695 (2003).

11. Davis, A. J., et al., J. Geophys. Res., 106, 29979-2998 (2001).

12. Cummings, A. C., Stone, E. C., and Steenberg, C. D., Astrophys. J., 578, 194-210 (2002).

13. Webber, W. R., McDonald, F. B., and Lukasiak, A., Astrophys. J., 599, 582-595 (2003).

14. Moskalenko, I. V., Strong, A. W., Mashnik, S. G., and J. E. Ormes, Astrophys. J., 586, 1050 (200).

15. Niebur et al., J. Geophys. Res., 108, No. A10, 8033, doi:10.1029/2003JA009876 (2003).

16. Gleeson, L. J., and Axford, W. I., Astrophys. J., 154, 1011, (1968).

17. Meyer, J. P., PhD thesis, University of Paris (Orsay), (1974)

18. Mewaldt, R. A., Stone, E. C., and Vogt, R. E., Astrophys. J., 206, 616-621 (1976).

19. Berghöfer, T. W.; Breitschwerdt, D., Astron. Astrophys., 390, 299-306 (2002). 